\title{
Analysis and Selection of insulating material based on 3D Printing PCB board
}

\author{
Bai Lei, Sun Changjian, Zhang Zhiyi* \\ Beihua University, Jilin, China, 132021 \\ ${ }^{*}$ Corresponding author
}

Keywords: Three dimensional printing; PCB manufacturing; insulation layer; electronic encapsulation.

\begin{abstract}
The traditional PCB plate makes process and adopts "reduction". The combination of art and technology has created a new "added material manufacturing" theory. There is important research value. The paper takes electrons, potting adhesive as insulation material of PCB circuit board. Printing tests for various types of potting compound which is carried out. The result of comparative data is obtained. Finally on the photo paper, the electronic circuit seal layer is completed as well as insulation performance test is conducted.
\end{abstract}

\section{Introduction}

Nowadays, with the development of technology, electronic products have already been developed. Ubiquitous in people's lives, as an electronic production, the demand for printed circuit boards is also increasing. Its quality requirements are also getting higher. Printed circuit board, hereinafter, referred to PCB, is electronic. Important electronic components in industrial products are used as electronic products which become the necessary substrate that is small to the calculator and electricity used in daily life. Products, which is used for watches, cell phones, computers, are great to communication and medicine. It involves in medical, military and other fields that is printed circuit boards. The traditional way to PCB production is mainly divided into reduction and processing.

There are two kinds of additive processing methods. At present, it is to reduce the processing method as the basic process PCB for manufacturers in China. The production cycle is long as well as the process is complicated. With the progress of the times, the cost of PCB products is increasing in China. The pollution of the environment is very serious, which is not in line with our country. but taking the road of sustainable development and cleaner production.3D printing is the technology of machining which is a kind of digital model in recent years. Type files are based on the use of powdered metals or plastics, even for bonding material and constructing objects by printing in layers.3D printing technology with the printed electronics technology has rapidly increased.

Combination is the hot topic of research. In terms of cost, 3D printing technology is processed by adding material method whose material is processed. The utilization ratio is higher than that of the traditional process while it is saving the cost. It is a process that cannot produce waste gas, waste liquid and cleaning at the same time.It is exactly process which is environmental protection, reduction of production steps and energy consumption. It is said that art is more efficient. In addition, for complex circuits, printing is usually desired.

The printing of multilayer circuits can be realized on the same substrate. The area of the substrate is greatly saved,at the same time, the circuit is small. Multilayer circuits is related to insulating materials between layers. The paper is based on the self-designed new 3D. The principle of the printer is borrowed.

According to the principle of FDM technology, which is based on this printer, we study multilayer electric circuit.

\section{PCB interlayer insulation material}

The PCB board described in this article is designed by 3D printing whose base selection for 
photographic paper is conductive .The silver paste is used as printing material. Through the slicing software, the printing path is planned, and finally passed. The nozzle is designed as well as extruded which is not printing process at normal temperature .It is printed by electronic circuits which need to be added. Solidification ,which is based on the electronic circuit of the first tier printing ,selects electricity. Sub potting adhesive is used as insulating material. Encapsulation glue which is known as electronic adhesive is widely used in the electronics industry. To protect electronic components by bonding, sealing is an indispensable and important insulating material.

According to the material, the sealant is mainly made of epoxy resin. There are two kinds of silicone resin glue circle as follow,

\subsection{Silicone encapsulating adhesive is based on organic polysiloxane}

With some crosslinking agents, catalysts, fillers, a potting material is made of auxiliaries. It has a wide range of using temperature range which is excellent thermal stability that is good conversion whose characters are stability. It is easy to shape and environmental protection that is excellent electrical insulation and certain water resistance.

\subsection{Epoxy encapsulating epoxy resin (EP) adhesive is a kind of epoxy resin}

It is compound with some curing agents and accelerators for grease based materials. Other auxiliaries have epoxy encapsulation. The adhesive has excellent adhesion whose electrical insulation and corrosion resistance which is corrosion property, high hardness, low curing shrinkage. The expansion system is linear. The number is small. However, the cured epoxy resins are brittle which is easy to crack. The high thermal expansion is coefficient. Heat resistance and heat transfer is low conductivity which is printed by insulating material.

\section{Flowability test}

\subsection{Printing material fluidity}

Liquidity, refers to the flow capacity of the fluid itself, is important criteria for physical properties .The technical specifications is the flow of fluid which is up to standard. For 3D printing PC, there is a plate insulation experiments. The fluidity of printed materials is whether or not the edge .Layer can be used to prepare the key to the materials that is formed. If printing materials is excessive liquidity, it will lead to the extrusion device passing through the nozzle. When the material is discharged, there is excess material flowing out .It must be the amount of extruded material. Too much materials, which result in 3D printing, can not control print out. Shape and accuracy, as well as materials are printed on the substrate.

Table 1 The physical nature of the insulating material

\begin{tabular}{|c|c|c|c|c|c|}
\hline models & HJ-101 & HJ-711 & HJ-721 & HJ-374 & HJ-375 \\
\hline color & $\begin{array}{c}\text { Translucent } \\
\text { liquid }\end{array}$ & $\begin{array}{c}\text { Translucent } \\
\text { liquid }\end{array}$ & White-liquid & Black-liquid & $\begin{array}{c}\text { Yellowish-brown } \\
\text { liquid }\end{array}$ \\
\hline density(g/cm) & 1.15 & 1.15 & 1.12 & 1.6 & 1.55 \\
\hline $\begin{array}{c}\text { Solidifying } \\
\text { type }\end{array}$ & normal & heat & normal & heat & heat \\
\hline permittivity & 2.9 & 3.2 & 2.9 & 4.0 & 4.2 \\
\hline temperture $\left(\mathrm{C}^{\circ}\right)$ & $-60-200$ & $-60-260$ & $-60-200$ & $-40-150$ & $-40-150$ \\
\hline
\end{tabular}

The flow of life is shaped if the flow of printed material is formed. Too weakness will result in extrusion device extrusion through the nozzle. The material is discontinuous, even it is printed on the substrate. Unable to extrude.

Five kinds of pouring sealant are selected through flowing sample.

Liquidity measurements are made to compare the suitability of each material, which is belonging 
to prints material, with the others by experiment.

The liquidity range is finally adjusted by two-component electrons.

The ratio of sealing glue makes the material reach the desired liquidity.

It is shown that the physical properties of the five kinds of electronic potting adhesive are clearly effective.

\subsection{Experiment on the fluidity of insulating materials}

The reasons that the insulating material is made of two component potting adhesive.

First, the insulation materials should be given according to each type of encapsulant which mixes evenly to ensure two kinds. The ingredients are mixed. Then the insulation materials will be prepared.

The second step will be measured in order to mold in the flow measurement. It is based on $100 \mathrm{~mm}$ as insulation for setting off the flow distance whose edge material is insulation. When the material enters the entrance of the channel from the center hole, it starts to time while the specified length is stopped. During the time, it is taken as a flow. In the same experimental environment, the streamline is simplified to level. The fluidity of printing materials is similar that it crosses flow gate. Under the condition of flow length, the length of the mobile $L$ and the arrival time are set. For the ratio of flow time for setting length is used to represent. Medium: the flow capacity of materials is that of flow time, i. e. silicone irrigation.

The time for sealing glue where goes into the flow path to the set length is Lilith distance which lays between silicone and potting sealant in spiral flow channel Leave.

In order to ensure the fluidity of insulating materials, they only have their own properties. The size of the gate is not related to the size of the runner. Insulation material, 1\#, 2\# mode in two channel sizes.

The results of experiments are compared with those obtained separately. If the error is within the allowable range, it can prove the insulation material. The mobility is independent of the size of the runner. Experiment number

Table 2 1\#Experiment on the mobility of the runner

\begin{tabular}{|c|c|c|c|c|c|}
\hline $\begin{array}{c}\text { models } \\
\text { items }\end{array}$ & HJ-101 & HJ-711 & HJ-374 & HJ-375 & HJ-721 \\
\hline Fluid distance L & $100 \mathrm{~mm}$ & $100 \mathrm{~mm}$ & $100 \mathrm{~mm}$ & $100 \mathrm{~mm}$ & $100 \mathrm{~mm}$ \\
\hline Fluid time $\tau$ & $235 \mathrm{~s}$ & $154 \mathrm{~s}$ & $323 \mathrm{~s}$ & $110 \mathrm{~s}$ & $142 \mathrm{~s}$ \\
\hline Fluid speed $\mathrm{v}$ & 0.42 & 0.65 & 0.31 & 0.91 & 0.70 \\
\hline $\begin{array}{c}\text { Experimental } \\
\text { temperature }\end{array}$ & $25 \mathrm{C}^{\circ}$ & $25 \mathrm{C}^{\circ}$ & $25 \mathrm{C}^{\circ}$ & $25 \mathrm{C}^{\circ}$ & $25 \mathrm{C}^{\circ}$ \\
\hline A team:B team & $10: 1$ & $1: 1$ & $3: 1$ & $3: 1$ & $2: 1$ \\
\hline
\end{tabular}

Table 3 2\# Experiment on the mobility of the runner

\begin{tabular}{|c|c|c|c|c|c|}
\hline $\begin{array}{c}\text { models } \\
\text { items }\end{array}$ & HJ-101 & HJ-711 & HJ-374 & HJ-375 & HJ-721 \\
\hline Fluid distance L & $100 \mathrm{~mm}$ & $100 \mathrm{~mm}$ & $100 \mathrm{~mm}$ & $100 \mathrm{~mm}$ & $100 \mathrm{~mm}$ \\
\hline Fluid time $\tau$ & $233 \mathrm{~s}$ & $155 \mathrm{~s}$ & $340 \mathrm{~s}$ & $110 \mathrm{~s}$ & $140 \mathrm{~s}$ \\
\hline Fluid speed $\mathrm{v}$ & 0.43 & 0.645 & 0.29 & 0.89 & 0.71 \\
\hline $\begin{array}{c}\text { Experimental } \\
\text { temperature }\end{array}$ & $25 \mathrm{C}^{\circ}$ & $25 \mathrm{C}^{\circ}$ & $25 \mathrm{C}^{\circ}$ & $25 \mathrm{C}^{\circ}$ & $25 \mathrm{C}^{\circ}$ \\
\hline A team:B team & $10: 1$ & $1: 1$ & $3: 1$ & $3: 1$ & $2: 1$ \\
\hline
\end{tabular}

\section{Insulation material printing experiment and result analysis}

Insulating materials can be made by designed extruded 3D printer itself. Material printing is done on photo paper, according to the first layer conductor.

The theoretical analysis of the layer is chosen by users. The calculation of resistance parameters continue to be selected. The metal that is separating sprinkler is used as the printing nozzle. The 
internal channel mechanism of the split precision nozzle is in the nozzle. The cross section changes at the cross section of the runner. The design of the sprinkler is gradual runner whose contraction still lays on surface. It ensures that a fluid does not generate in the nozzlewith a certain viscosity . The tendency tis to separate from the wall. And the sprinkler is installed in the autonomy.

Printing is done on the extrusion mechanism of the designed 3D printer, because the five insulating materials are selected from the insulation layer whose printed materials is conductive silver paste which is compared with most of them whose edge material has better fluidity than conductive silver paste. When it chooses straight. The nozzle with a diameter of $03 \mathrm{~mm}$ ensures the printing accuracy of insulating material that is setting the printed 3D graphics for a the data as followed ,its length is $60 \mathrm{~mm}$, the heigh is $02 \mathrm{~mm}$, width is $0.6 \mathrm{~mm}$. All the datas have three parallel lengths of $2 \mathrm{~mm}$.

The cube is printed.

Table 4 Related parameters of insulating materials

\begin{tabular}{|c|c|c|c|c|c|}
\hline $\begin{array}{c}\text { models } \\
\text { items }\end{array}$ & HJ-101 & HJ-711 & HJ-374 & HJ-375 & HJ-721 \\
\hline A team:B team & $10: 1$ & $1: 1$ & $3: 1$ & $3: 1$ & $2: 1$ \\
\hline Model distance(mm) & Nothing & 6 & 2 & 4 & 7 \\
\hline Nozzle size(mm) & Nothing & $0.3 / 0.4$ & $0.4 / 0.7$ & $0.4 / 0.7$ & $0.3 / 0.4$ \\
\hline Step number per mm & 1500 & 1000 & 1400 & 1200 & 1000 \\
\hline
\end{tabular}

Table 5 Curing time and actual molding dimensions of insulating materials

\begin{tabular}{|c|c|c|c|c|c|}
\hline models & HJ-101 & HJ-711 & HJ-374 & HJ-375 & HJ-721 \\
\hline items & Normal & Heat & Heat & Heat & Heat \\
\hline $\begin{array}{c}\text { Curing type } \\
\text { temperature(C })^{\circ}\end{array}$ & 30 & 100 & 100 & 100 & 100 \\
\hline Curing time(min) & 40 & 30 & 70 & 50 & 40 \\
\hline Trim length(mm) & Nothing & 57 & 58 & 58 & 55 \\
\hline Trim width(mm) & Nothing & 13.5 & 5.9 & 9.8 & 17 \\
\hline $\begin{array}{c}\text { Theoretical } \\
\text { width(mm) }\end{array}$ & 5.8 & 13.8 & 5.8 & 9.8 & 15.8 \\
\hline Thickness(mm) & Nothing & 0.74 & 0.84 & 1.19 & 0.82 \\
\hline
\end{tabular}

\section{Conclusion}

This paper is based on the self designed extruded 3D printer. The photographic paper is a substrate, while the conductive silver paste is the conductor layer. The premise is in the five selected categories (HJ-101, HJ-711).HJ-721, HJ-374, HJ-375) experimental specification for potting adhesive the flow test .The insulation layer printing are carried out respectively. Considering the analysis, H-101 can not pass the printer. HJ-721 can be played through printers. Printing, but the accuracy and molding effect, are not satisfied with insulation layer. HJ-374 is not environmentally friendly and has long curing time.

Printing time will block nozzle as well as the cycle is long. Unfit for action, the printing material of the printer insulation layer. H-375 can be used as printing material .It has low shrinkage rate and small error, but after curing, the insulation layer is crisp and hard, which is not allowed to bend at HJ-711that is most suitable for printing the insulating layer of this printer.

The material is used as certain flexibility after curing. PCB boards have wide application prospects in daily life. We need further research and development.

\section{Acknowledgements}

This paper is "Science and technology project of Jilin education department in $13^{\text {th }}$ Five-Year" Project Name: Research and application of igh precision control strategy for 3D ptinter 
Project Code: JJKH20180339KJ

\section{References}

[1] Lei Yu. Analysis of cleaner production status in printed circuit board industry Research on evaluation method D Huazhong University of Science and Technology, 2012

[2] Yang Enquan The influence of 3D printing technology on the development of aviation manufacturing industry Sound aviation science and technology, 2013 (01): 13-17

[3] Gao Yu Le, Shi Changchun, Dong Dechao, Chen Rong, Dan Bin, based on 3D. Research on Rapid Prototyping Technology of flexible electronic circuits for printing technology Gate printed circuit information, 2016,24 (03): 5-8+23.

[4] Patrick F. Flowers, Christopher Reyes, Shengrong Ye, Myung Jun Kim, Benjamin J. Wiley. 3D printing electronic components and Circuits with conductive thermoplastic Filament[J]. Additive Manufacturing, 2017, 18

[5] Zhao Nian, heat conduction and halogen free flame retardant modification of [D] Southern Polytechnic University, 2015

[6] Zhou De Jian.3D printing technology and its manufacture in electronic products Discussion on the application of [A] electronic mechanics in China Electronics Society

[7] Lei Weihua, Zhu Jingzhi, Xu Bing plus liquid silicone rubber. Study on fluidity and structure properties [J material guide Newspaper, 2015,29 (12): 89-95

[8] Zhou Zhiming, Song Xiaofang, Chen Yuanfang, Tang Liwen, Liu Chun Xiao Zhipei Research on metal mobility testing devices And technology, 2014,12 (05): 1-2+7

[9] Zhao Yazhong, Cai Guangyu, Zhang Xin, ban Ya Feng liquidity test gold Design and application of generic mold [J hot working worker 